\title{
Síndrome Peutz Jeghers y Obstrucción Intestinal. Caso Clínico
}

\author{
CAROLINA DONOSO C. ${ }^{1}$, OSCAR CARVAJAL F. ${ }^{2}$
}

1. Cirujano Pediatra. Servicio de Cirugía Pediátrica. Hospital Clínico San Borja Arriarán. Instructor, Departamento de Pediatría y Cirugía Infantil. Facultad de Medicina. Universidad de Chile.

2. Cirujano Pediatra. Servicio de Cirugía Pediátrica. Hospital Clínico San Borja Arriarán. Profesor Asistente, Departamento de Pediatría y Cirugía Infantil. Facultad de Medicina. Universidad de Chile.

\begin{abstract}
Peutz Jeghers Syndrome and Bowel Obstruction in Children: A Clinical Case

Peutz-Jeghers Syndrome (PJS) is an infrequent genetic disorder characterized by intestinal hamartomas and characteristic mucocutaneous pigmentation. It is frequently the cause of intususception, intentinal obstruction and/or intestinal bleeding. An 8 y.o. patient is described who presented with bowel obstruction and a history of familiar PJS polyposis. Exploratory laparotomy showed that the obstruction was due to a mass of sizable polyps which obstructed the intestinal lumen in the jejunum and colon. Surgical treatment consisted of double intestinal resection and termino-terminal anastomosis. This procedure resolved the acute intestinal obstruction and saved the patient's life.
\end{abstract}

(Key words: Peutz-Jeghers, polyposis, intestinal obstrucion).

Rev Chil Pediatr 2010; 81 (5): 437-444

\section{RESUMEN}

El síndrome de Peutz-Jeghers (SPJ) es una enfermedad genética poco frecuente, caracterizada por la presencia de pólipos hamartomatosos intestinales y pigmentaciones mucocutáneas características ${ }^{1}$. Con frecuencia es causal de intususcepción, obstrucción intestinal y/o hemorragias digestivas. Se describe el caso clínico de una paciente de 8 años que tenía el diagnóstico de poliposis familiar de Peutz Jeghers y que presentó obstrucción intestinal. En la laparotomía exploradora se constató que la obstrucción intestinal era secundaria a una masa de grandes pólipos sésiles, que obstruían el lumen intestinal en yeyuno y colon. El tratamiento quirúrgico consistió en doble resección intestinal y anastomosis termino-terminal. Este procedimiento quirúrgico constituyó una solución al cuadro agudo de obstrucción intestinal de la paciente, siendo de vital importancia para mantener su sobrevida.

(Palabras clave: Peutz-Jeghers, poliposis, obstrucción intestinal).

Rev Chil Pediatr 2010; 81 (5): 437-444

Trabajo recibido el 14 de diciembre de 2009, devuelto para corregir el 10 de febrero de 2010, segunda versión el 03 de septiembre de 2010, tercera versión el 28 de septiembre de 2010, aceptado para publicación el 29 de septiembre de 2010.

Correspondencia a:

Dra. Carolina Donoso C.

E-mail:dra.donoso@gmail.com 


\section{Introducción}

El Síndrome de Peutz-Jeghers (SPJ), es un síndrome poco frecuente, de herencia autosómica dominante, no ligado al sexo, que afecta tanto a hombres como a mujeres. La enfermedad fue reconocida por vez primera en 1921 por Peutz en una familia holandesa, siendo la publicación de Jeghers 28 años después en un paciente que falleció debido a una invaginación intestinal complicada. Se caracteriza por pigmentaciones puntiformes en mucosa oral, labios, región palmar y plantar; y en forma ocasional, en la región perianal. El SPJ está asociado con un riesgo mayor de malignización gastrointestinal y de otros sitios. Existen numerosos reportes de cáncer en pacientes con SPJ y la distribución de los cánceres en el tubo digestivo es similar a la de los pólipos hamartomatosos.

El objetivo de nuestro trabajo es mostrar el caso clínico de una paciente con síndrome de Peutz Jeghers que desarrolló varias complicaciones, principalmente desde el punto de vista quirúrgico. En la literatura nacional encontramos sólo dos reportes de esta enfermedad: un estudio familiar, que abarca a un padre y su hija de 5 años, pero que nunca ha presentado este nivel de complicaciones y un caso clínico en un paciente adulto ${ }^{1,2}$. La gran mayoría de los pacientes descritos internacionalmente corresponden a pacientes adultos y no existen reportes de pacientes pediátricos que hayan sido sometidos a una cirugía de estas características.

\section{Caso clínico}

Paciente de 8 años, de sexo femenino, con antecedentes de trastorno de conducta alimentaria (anorexia alternado con episodios de bulimia) y padre con poliposis de Peutz Jeghers, fallecido por cáncer esofágico a los 48 años.

Debido a estos antecedentes y porque clínicamente la niña presentaba máculas mucocutáneas características de poliposis en labios (figura 1), la paciente se controlaba en el policlínico de gastroenterología de nuestro hospital desde los 6 años de edad. Dentro de sus estudios destacaba una endoscopía digestiva alta (mayo 2008) que evidenciaba múltiples pólipos gástricos hamartomatosos, antrocorporales Yamada I. Se planteó entonces, el diagnóstico de poliposis de Peutz Jeghers y se completó el estudio con una endoscopía digestiva baja, encontrándose también múltiples pólipos en colon ascendente Yamada IV, sin signos de atipia celular. En su historia clínica no había antecedentes de resección de pólipos vía endoscópica, ni tampoco antecedentes de habérsele practicado un tránsito intestinal.

El cuadro quirúrgico se caracterizó por dolor abdominal, difuso, cólico, que aumentaba en intensidad. Fue evaluada tanto en su consultorio como en el hospital, no impresionando un cuadro quirúrgico y se manejó sintomáticamente. Sin embargo, el dolor persistió y se agregó anorexia y vómitos ocasionales. Consultó nuevamente, intentándose un tratamiento similar, pero el dolor se hacía cada vez más intenso. Finalmente, a los 7 días la paciente comenzó con deposiciones con sangre fresca y dolor cólico muy intenso, por lo que consultó nuevamente.

Ingresó al hospital muy decaída, con compromiso del estado general, lengua saburral, y pigmentación característica en mucosa bucal y labios. Al examen físico destacaba, que el abdomen no se encontraba distendido, pero sí estaba muy doloroso a la palpación, y se palpaba una masa en el hemiabdomen superior. Clínicamente se diagnosticó una obstrucción intestinal, probablemente secundaria a una

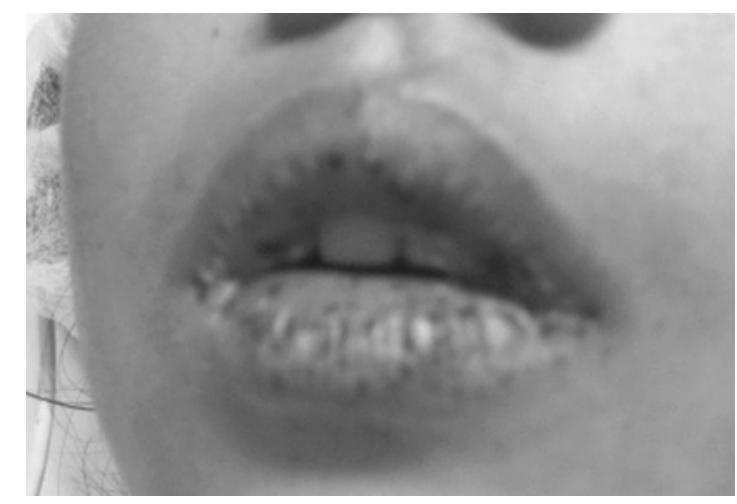

Figura 1. Pigmentación mucocutánea en labios, característico de poliposis de Peutz-Jeghers. 
invaginación intestinal y se solicitó inmediatamente una ecografía abdominal, para confirmar el diagnóstico. La ecografía abdominal informó "extensa invaginación intestinal que compromete íleon, colon derecho, transverso e izquierdo. Signos imagenológicos sugerentes de asas comprometidas" (figura 2).

Con este diagnóstico ecográfico, más la alta sospecha clínica, se decidió la laparotomía exploradora. Vale la pena mencionar que debido a varios antecedentes (que serán tratados en la discusión) no era recomendable una reducción neumática o hidrostática. Se realizó una laparotomía transversa paraumbilical derecha, se realizó una apertura longitudinal de la vaina de los rectos, lo que permitió un acceso amplio a la cavidad. Se observó intestino delgado con gran distensión de asas de yeyuno y se palpó un conglomerado de masas semi-móviles en su interior. A nivel del colon ascendente se encontró una zona sugerente de haber tenido una invaginación intestinal, con una masa intra luminal de similares características a las del yeyuno. Se realizó entonces una enterotomía, la que objetivó múltiples masas polipoídeas sésiles, de gran base, no pediculadas, que obstruían el lumen intestinal a nivel de yeyuno, y también en colon ascendente y transverso. (figuras 3 y 4). Se efectúo la resección intestinal de las zonas afectadas, resecando $15 \mathrm{~cm}$ de yeyuno y realizando una hemi-colectomía de colon ascendente y transverso. Se realizó una anastomosis término-terminal yeyunoyeyunal; y una anastomosis termino-terminal colo-colónica. Finalmente, se realizó la apendicectomía profiláctica.

Durante el postoperatorio la paciente presentó una buena evolución. Tuvo algo de dolor abdominal en los primeros días postoperatorios y leve distensión abdominal inicial, todo lo cual estuvo dentro de lo esperable. Se indicó régimen $0, \mathrm{SNG}$ a caída libre y alimentación parenteral. Inició régimen hídrico al $7^{\circ}$ día y la alimentación parenteral se suspendió al $9^{\circ}$ día. La sonda nasogástrica instalada presentaba residuo bilioso al inicio y luego contenido

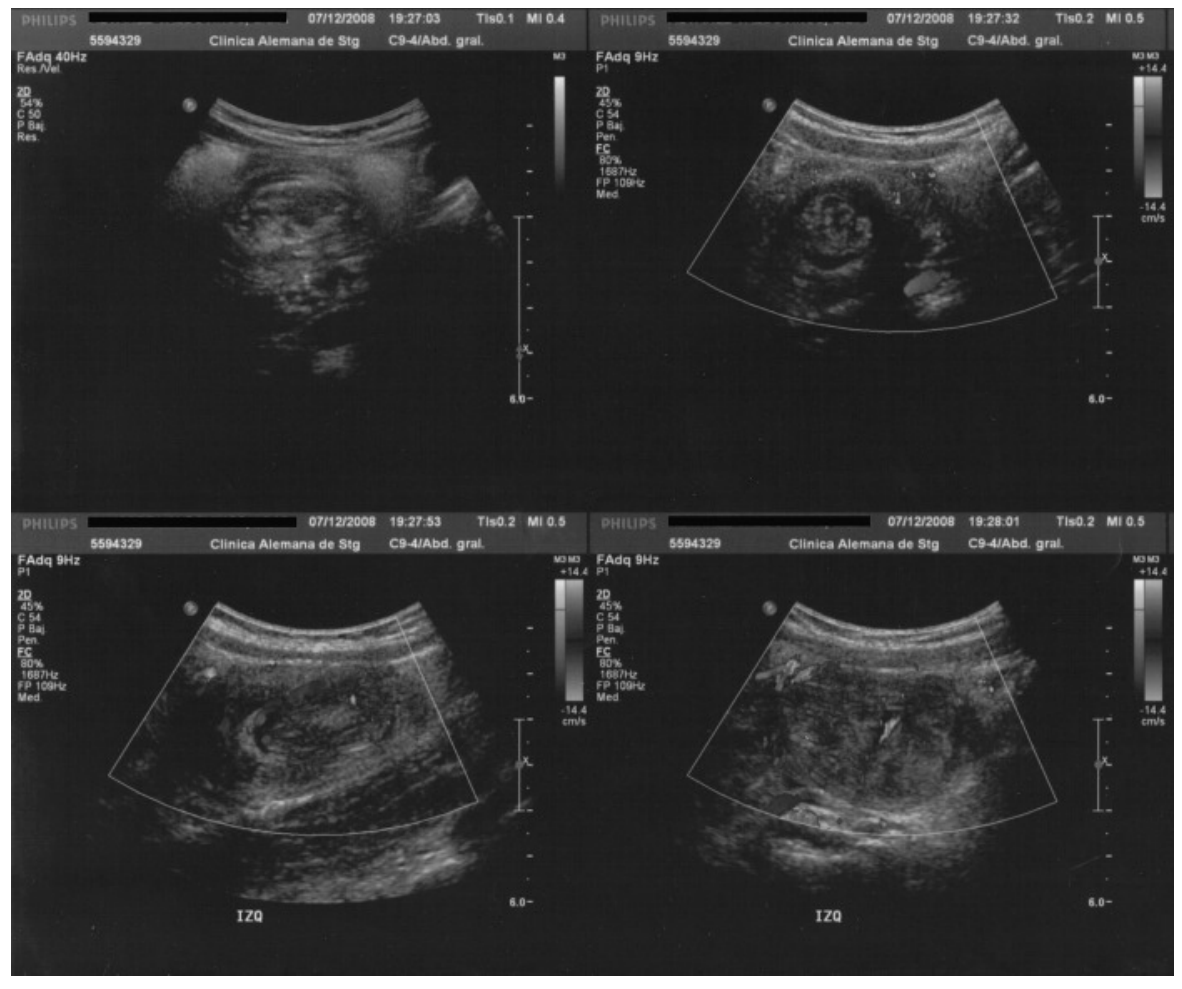

Figura 2. Eco Abdominal: muestra imagen sugerente de extensa invaginación intestinal. 


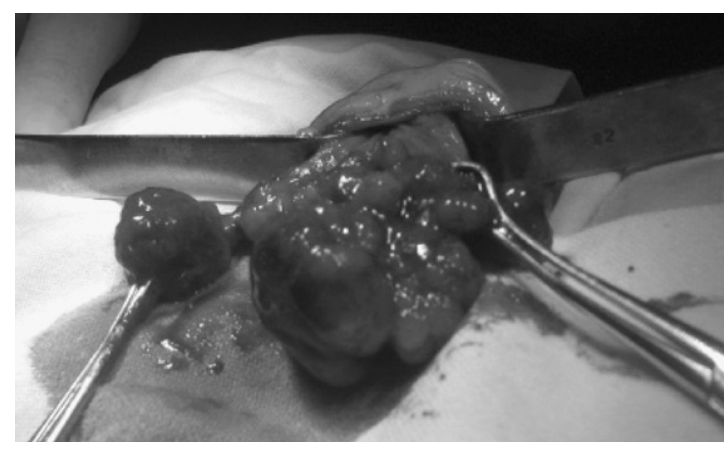

Figura 3. Obsérvese masa polipoidea que protruye a través del lumen intestinal, en forma de racimo de uva y ocluyendo todo el lumen intestinal.

claro, hasta que finalmente disminuyó su débito logrando ser retirada al $6^{\circ}$ día sin incidentes. Al realimentarse completamente con régimen liviano la paciente presentó buena tolerancia. No tuvo vómitos, ni tampoco dolor abdominal. Completó 10 días de tratamiento antibiótico triasociado (Metronidazol, Amikacina, Penicilina Sódica).

Se dio de alta a los 12 días de haber sido operada, se fue a su domicilio en buenas condiciones generales y su seguimiento ambulatorio ha sido satisfactorio. Actualmente, 1 año 8 meses post operada, se encuentra sin dolor abdominal, alimentándose sin problemas. No ha tenido nuevos cuadros de obstrucción intestinal.

\section{Discusión}

El síndrome de Peutz-Jeghers (SPJ) es una enfermedad autosómica dominante con un alto grado de penetrancia para poliposis y pigmentación cutánea; siendo su frecuencia similar en hombres y mujeres y que varía de 1/60 000$1 / 300000$ individuos $^{3}$.

El gen responsable de éste síndrome ha sido mapeado en el cromosoma 19p13.3 y el defecto en las familias afectadas involucra mutaciones en un gen que codifica una kinasa serina treonina ${ }^{4}$. Otras observaciones sugieren que existe un segundo locus, probablemente localizado en el cromosoma $13^{4,5}$.

En un principio se consideró al SPJ como

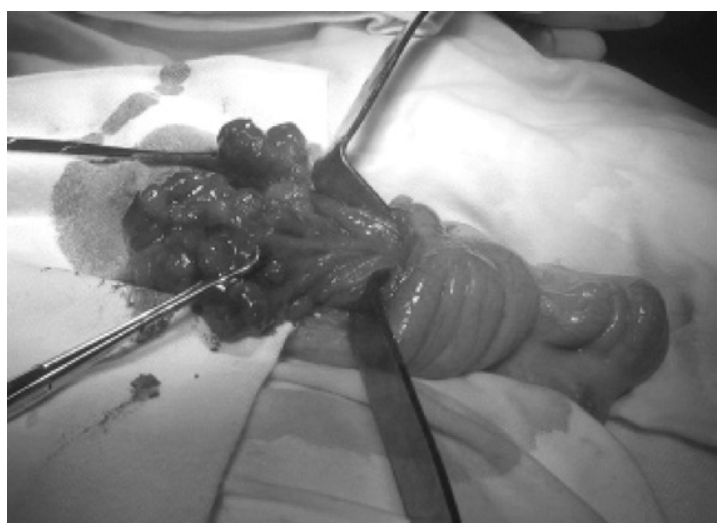

Figura 4. Visión lateral de pólipos en lumen intestinal.

una enfermedad hamartomatosa benigna, actualmente se sabe que los pacientes con SPJ tienen un riesgo de cáncer 15 y 18 veces mayor que el de la población general tanto para desarrollar un cáncer gastrointestinal como extraintestinal ${ }^{6-8}$. Por ello se recomienda extraer los pólipos cuando éstos son visualizados. Lo ideal es realizar, en todos aquellos pacientes portadores de SPJ, screening de enfermedades malignas en forma periódica; mamografía y PAP en las mujeres, ecografía y control con antígeno carcinoembrionario en el hombre.

Considerando que el SPJ tiene un mecanismo de herencia autosómico dominante, se debe tener también un alto índice de sospecha en los familiares de primer grado, ya que estos tienen un $50 \%$ de probabilidades de poseer esta mutación. Nuestra paciente tiene el antecedente de padre con poliposis familiar, por lo que en ella el seguimiento a largo plazo y screening de posibles neoplasias será algo primordial.

Las manifestaciones clínicas del SPJ son dos: 1) Máculas pigmentadas mucocutáneas y 2) Múltiples pólipos hamartomatosos gastrointestinales, los cuales son benignos, pero pueden tener una transformación maligna. Actualmente, la OMS exige cuatro requisitos posibles para hacer el diagnóstico: 1) La presencia de tres o más pólipos de Peutz-Jeghers confirmados histológicamente; 2) Cualquier número de pólipos de Peutz-Jeghers con historia familiar de SPJ; 3) Pigmentación mucocutánea característica, con historia familiar de SPJ, y 4) Cualquier número de pólipos de 
Peutz-Jeghers y pigmentación mucocutánea característica ${ }^{9}$. Nuestra paciente cuenta con todos los criterios.

Los pólipos pueden variar en su tamaño y localización; se localizan principalmente en el intestino delgado (65\%), y pueden también estar presentes en el colon $(30 \%)$, recto y estómago ${ }^{3,4,10}$. Esta paciente presentaba pólipos intestinales en colon ascendente, transverso y yeyuno; además de los pólipos gástricos encontrados en la endoscopía anterior.

Existen recomendaciones para la evaluación de pacientes con SPJ que han sido propuestas por la Asociación de Gastroenterología y que se resume en la siguiente tabla (tabla 1$)^{1 .}$

Como parte del estudio de pacientes con SPJ y los familiares de primer grado (padres, hermanos e hijos) se debe tener una anamnesis dirigida a la búsqueda de episodios de dolor abdominal, hemorragia digestiva y anemia. En el examen físico, buscar manchas pigmentadas en la mucosa oral, labios, párpados, dedos y región perianal.

En cuanto al estudio imagenológico se debe contar con una ecografía abdominal, tomografía axial computada y una radiografía de tránsito intestinal para pesquisar pólipos del intestino delgado. En esta patología el rol que juega la panendoscopía alta y la colonoscopía larga como apoyo diagnóstico y terapéutico es fundamental, ya que se tiene acceso a los pólipos de estómago, duodeno y colon, pudiendo extraerse los de mayor tamaño ${ }^{11}$. El $70 \%$ de los pacientes colectomizados por poliposis del colon, tienen además pólipos en duodeno, y de los pacientes con cáncer gastrointestinal $30-40 \%$ tienen pólipos duodenales ${ }^{11,12}$. Poco se sabe, en cambio, de la poliposis de yeyuno e íleon, pero existe una correlación entre los pólipos de yeyuno proximal y pólipos de duodeno, lo que implica peor pronóstico y mayor riesgo de malignización; principalmente por el sub diagnóstico de estas tumoraciones debido a lo invasivo de los exámenes necesarios para detectarlos. Nuestra paciente, por ejemplo, tenía identificados pólipos en el estómago y colon, pero no había nada descrito respecto a la masa polipoídea en yeyuno, que finalmente la llevó a obstrucción intestinal. De ahí la necesidad de un screening del tracto digestivo superior. Los estudios actuales ${ }^{13}$ recomiendan $\mathrm{Rx}$. tránsito intestinal 2 veces al año desde los 10 años de edad, lo que es en extremo difícil debido a la constante exposición a radiación ionizante. Nuestra paciente es menor de 10 años, por lo que la decisión de realizar este examen es aún más controversial. Probablemente dados los antecedentes importantes del cáncer y más aún fallecimiento del padre, además de la visualización endoscópica de sus pólipos en estómago, debería haberse planteado anteriormente para prevenir este tipo de complicaciones.

En la actualidad cobra una especial importancia la enteroscopía de doble balón. Esta es una nueva técnica endoscópica que sirve para diagnosticar enfermedades del intestino delgado que antes con la simple endoscopía o colonoscopía no podían diagnosticarse. El enteroscopio tienen dos balones en la punta (figura 5) que se van inflando y desinflando mediante un equipo manométrico, el cual controla la presión de los balones para permitir el avance y también plegando sobre sí mismo el intes-

Tabla 1. Recomendaciones de Asociación de Gastroenterología de seguimiento y screening en pacientes SPJ

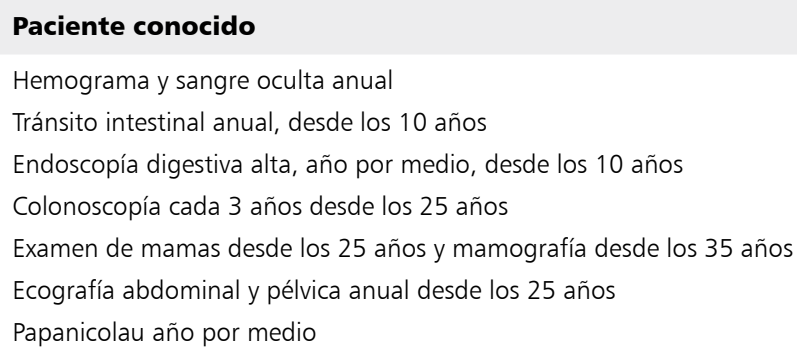

\section{Familiar asintomático}

Hemograma anual

Tránsito intestinal a los 12,18 y 24 años 


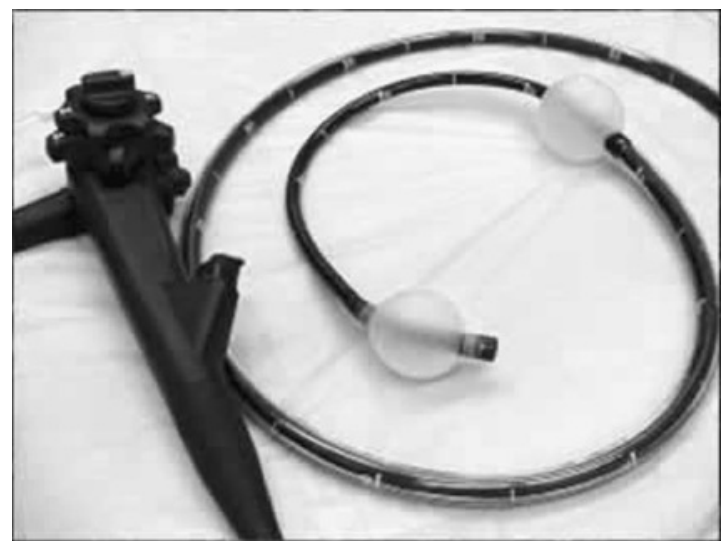

Figura 5. Enteroscopio de doble balón, para ir avanzando a través del intestino delgado.

tino delgado ${ }^{14}$. En la mayoría de los casos se consigue explorar del 70 al $90 \%$ del intestino delgado con esta técnica. Además se pueden realizar biopsias, polipectomías coagulación, etc $^{15}$. Pero aún no está disponible en todos los medios.

Lo óptimo sería la RNM intestinal, que es cara, e inalcanzable en forma rutinaria en nuestro medio. Otro método útil en el diagnóstico es el recuento de glóbulos rojos para los pacientes portadores de poliposis del intestino delgado, para objetivar posibles sangrados o complicaciones.

Las principales causas de morbi-mortalidad en SPJ son: 1) Obstrucción intestinal; 2) Invaginación intestinal $(43 \%)$; que en la mayoría de los casos coexisten, debido a la invaginación intestinal secundaria a pólipo; 3) Dolor abdominal recurrente $(23 \%)$; 4) Hematoquezia $(14 \%)$ y 5) Prolapso rectal del/los pólipos $(7 \%)^{4,16}$. Esto es de vital importancia a la hora de hacer el diagnóstico, ya que la principal complicación y causa de morbi-mortalidad del SPJ es la obstrucción intestinal. Nuestra paciente tenía una clara clínica de obstrucción intestinal, secundaria a pólipos intestinales, sin embargo, no fue hasta la tercera consulta en que esto fue diagnosticado. Además estos pacientes tienen la característica de "avisar" el diagnóstico (pigmentación en labios), por lo que es imprescindible pensar en esta entidad al enfrentarse a un paciente con estas características, a fin de evitar complicaciones como la resección intestinal amplia que debió realizarse en esta paciente.

Se realizó una ecografía abdominal, lo que está dentro de los exámenes de elección por una invaginación intestinal como la causa de la obstrucción. En la ecografía se observó una imagen sugerente de invaginación intestinal completa, desde el íleon hasta el colon transverso, con signos de compromiso de asas.

El tratamiento de SPJ consiste en la resección de los pólipos grandes ( $>5 \mathrm{mms}$ ) o pólipos hemorrágicos, preferentemente mediante una colonoscopía. La laparotomía y resección intestinal debe ser reservado para las invaginaciones intestinales recurrentes, la obstrucción intestinal secundaria a pólipos o la hemorragia digestiva persistente. Esta es la razón de que nuestra paciente no pudiese haber sido abordada con una reducción neumática o hidrostática. Estos procedimientos pueden ser realizados en cuadros de invaginación intestinal, sólo bajo ciertas circunstancias: Invaginación por primera vez, en menores de 2 años, sin una guía o cabeza de la invaginación y con menos de 12 a 24 hrs de evolución. Nuestra paciente no cumple con estas características desde ningún aspecto. Claramente tenía una guía de invaginación (pólipos), llevaba alrededor de 7 días con el diagnóstico y con signos de sufrimiento intestinal en la ecografía. De haberse abordado de una manera "conservadora" corría el riesgo de perforación intestinal.

En este caso al realizar la cirugía se observó efectivamente el compromiso de asas y no se encontró la invaginación intestinal descrita en la ecografía. Había signos de obstrucción intestinal, con dilatación de asas; y en dos segmentos intestinales (yeyuno distal, colon ascendente y transverso), se encontró una masa de pólipos intestinales, no pediculados en racimo de uva, que obstruían completamente la luz intestinal; siendo la causa de la obstrucción. Se decidió la resección de ambos segmentos (yeyuno y colon ascendente y transverso) y la posterior anastomosis término-terminal.

El tratamiento quirúrgico del SPJ es fundamental, ya que no sólo evita las complicaciones ya descritas, secundarias a los pólipos, sino que disminuye el riesgo de cáncer producto de la malignización de los pólipos. Esta niña 
no es operada hasta el $7^{\circ}$ día de su evolución, condicionando posiblemente su resección intestinal. Influye también que, debido al gran compromiso del estado general, labilidad de la paciente y complejidad de realizar 2 resecciones amplias simultáneas ( $5 \mathrm{hrs}$ de cirugía), no fuera posible realizar una exploración de otras localizaciones del intestino, buscando más pólipos de difícil diagnostico menos invasivo.

Los últimos estudios ${ }^{9}$ incluso han planteado soluciones quirúrgicas radicales, como por ejemplo, colectomía con anastomosis ileo-rectal o la proctocolectomía con anastomosis ileoanal. Aún no hay un consenso sobre cuál de ellas sería la de elección. No se ha comunicado mayor riesgo significativo de cáncer en aquellos pacientes con remanente de recto, pero si se ha visto mejor calidad de vida en aquellos con ileo-recto anastomosis ${ }^{17}$. Ambas están indicadas en pacientes con poliposis severa y al ser la poliposis de Peutz Jeghers una adenomatosis familiar hay un gran porcentaje de pacientes jóvenes y asintomáticos, por lo que la decisión quirúrgica no siempre es fácil. Es la única conducta realmente efectiva de disminuir al máximo el riesgo de malignización. En esta paciente se efectuaron dos resecciones intestinales completas, debido a la complicación evidente (obstrucción), pero también considerando el antecedente del padre con poliposis familiar, quien falleció por un cáncer gastrointestinal (esófago).

\section{Conclusión}

Esta no es la presentación típica del SPJ y obstrucción intestinal. Lo más frecuente es la obstrucción intestinal secundaria a la invaginación del intestino, pero ésta es una presentación que debe tenerse en cuenta, ya que los pacientes con este diagnóstico tienen una amplia gama de eventos que pueden surgir a raíz de estos pólipos. El ideal habría sido contar con el antecedente de los pólipos en yeyuno, y el haber realizado un estudio más acabado de todos los segmentos del intestino; además de un diagnóstico más precoz en la primera consulta. Esto probablemente habría evitado una resección intestinal tan amplia, o habría permitido la extracción de aquellos pólipos colónicos mediante la colonoscopía.

Actualmente la paciente no se encuentra con signos de intestino corto y ha estado asintomática desde entonces, pero es crucial mantener un estudio y seguimiento estricto para no incurrir en nuevas resecciones amplias de intestino.

\section{Referencias}

1.- Guzmán $S$, Nervi F, Llanos $O$, et al: Impaired liquid clearence in patients with previous acute pancreatitis. Gut 1985; 26: 888-91.

2.- Grez M, Prado R, Lahsen J, Hernández J: Síndrome de Peutz-Jeghers complicado. Reporte de un caso. Rev Chil Cir 2008; 60: 249-54.

3.- Ferreiro M, Harris P, Larrain F, Duarte I, Repetto G: Una familia con síndrome de Peutz-Jeghers. Rev Chil Pediatr 2000; 71: 214-9.

4.- Hutchinson J: Pigmentation of the lips and mouth. Arch Surg 1896; 7: 290.

5.- Duchini A, Carethers J: Contributor Information and Disclosures. Peutz-Jeghers Syndrome: eMedicine Gastroeneterology. Updated: Jan 4, 2007. http://emedicine. medscape.com/article/182006-overview

6.- Schreibman I, Baker M, Amos C, McGarrity TJ: The Hamartomatus Polyposis Syndromes: A Clinical and Molecular Review. Am J Gastroenterol 2005; 100 (2): 476-90.

7.- Azis $O$, Athansiou T, Fazio VW, et al: Meta-analysis of observational studies of ileorectal versus ileal pouchanal anastomosis for familial adenomatous polyposis. Br J Surg 2006; 93 (4): 407-17.

8.- Giardiello FM, Welsh SB, Hamilton SR: Increased risk of cancer in the Peutz-Jeghers syndrome. N Engl J Med 1987; 316: 1511-4.

9.- Boardman LA, Pittelkow MR, Couch FJ, et al: Association of Peutz-Jeghers-like mucocutaneous pigmentation with breast and gynecologic carcinomas in women. Medicine (Baltimore) 2000; 79: 293-8.

10.- World Health Organization Classification of Tumors: Pathology \& Genetics. Tumors of the digestive system 2000: 74.

11.- Gruber S, Entius M, Petersen G, et al: Pathogenesis of adenocarcinoma in Peutz-Jeghers syndrome. Cancer Res 1998; 58 (23): 5267-70. [Medline].

12.- Schulmann K, Hollerbach $S$, Kraus $K$, et al: Feasibility and Diagnostic Utility of Video Capsule Endoscopy for the Detection of Small Bowel Polyps in patients 
DONOSO C. y cols.

with Hereditary Polyposis Syndromes. Karsten Am J Gastroenterol 2005; 100: 27-37.

13.- Foley TR, McGarrity T, Abt BA: Peutz-Jeghers syndrome: A clinicopathologic survey of the "Harrisburg Family" with a 49-year follow up. Gastroenterology 1988; 95: 153-4.

14.- Boardman L, Thibodeau S, Schaid D, et al: Increased risk for cancer in patients with the Peutz-Jeghers syndrome. Ann Intern Med 1998; 128 (11): 896-9. [Medline].

15.- Moreira V, López R: Enteroscopía de doble balón. Rev Esp Enferm Dig [online] 2008; 100 (1): 57-9.

16.- http://www.endoscopiauc.cl/2009/12/enteroscopia-dedoble-balon-mayor-precision-en-el-estudio-y-tratamiento-del-intestino-delgado.
17.- Wu Y, Tsai C, Yang J, Hwang MH: Gastroduodenal intussusception due to Peutz-Jeghers syndrome. A case report. Hepatogastroenterology 1994; 41 (2): 134-6.

18.- Giardiello F, Welsh S, Hamilton $S$, et al: Increased risk of cancer in the Peutz-Jeghers syndrome. N Engl J Med 1987; 316 (24): 1511-4. [Medline].

19.- Fernández M, Martinez M, Fernández J, Trabazo S, Gamborino E, Forteza J: Peutz-Jeghers syndrome in a neonate. J Pediatr 1995; 126: 965-7.

20.- Wu Y, Tsai C, Yang J, Hwang MH: Gastroduodenal intussusception due to Peutz-Jeghers syndrome. A case report. Hepatogastroenterology 1994; 41 (2): 134-6.

21.- Pinto J, Rebaza S, Muñoz S, Maco V: Síndrome de Peutz-Jeghers y Adenocarcinoma de colon. Rev Gastroenterol Perú 2004; 363-7. 